\title{
VON NEUMANN REGULAR RINGS: CONNECTIONS WITH FUNCTIONAL ANALYSIS
}

\author{
BY K. R. GOODEARL
}

Most ring theorists and functional analysts are at least vaguely aware that von Neumann invented regular rings 45 years ago in connection with certain operator algebras, but that connection has grown rusty and pretty much disused as regular rings and operator algebras have gone their separate ways. My purpose here is to report on several more recent connections between regular rings and functional analysis, developed in the past decade, through which each subject has made a positive contribution to the other.

The first three sections of this report are ancient history, sketching the original development of regular rings in connection with continuous geometries, von Neumann algebras, and $A W^{*}$-algebras, and providing some of the relevant ring and operator algebra concepts for those readers who don't have them right at hand. The remaining three sections sketch the recent connections, including some of the ways in which regular rings have aided the study of Rickart $C^{*}$-algebras and approximately finite-dimensional $C^{*}$-algebras, and some of the ways in which Choquet simplices have aided the study of regular rings.

I. Complemented modular lattices and regular rings. Regular rings were invented by von Neumann in the mid-1930's in order to provide an algebraic framework for studying the lattices of projections in the operator algebras he was investigating. This framework actually dealt with a remarkably large class of general lattices, including most complemented modular lattices, which were then thought to be the appropriate setting for the logical formalism of quantum mechanics.

Von Neumann modelled this framework on the coordinatization of classical projective geometry, which was at that time just being recast in a lattice-theoretic mold, by Birkhoff [5] and Menger [18], among others. These authors viewed projective geometries as lattices $L$ satisfying

(a) Complementation. The lattice $L$ has a least element 0 and a greatest element 1 , and every element $x \in L$ has at least one complement, namely an element $y \in L$ such that $x \wedge y=0$ and $x \vee y=1$.

(b) Modularity. Whenever $x, y, z \in L$ with $x \leqslant z$, then $(x \vee y) \wedge z=$ $x \vee(y \wedge z)$. (This is a weak form of the distributive law.)

This paper is an expanded version of an hour address given on January 3, 1980 at the Annual Meeting of the American Mathematical Society in San Antonio, Texas; received by the editors April 20, 1980.

1980 Mathematics Subject Classification. Primary 16A30, 06C20, 46L10, 46L05, 46A55. 
(c) IRREDUCIBILITY. The only elements in $L$ with unique complements are 0 and 1. (This prevents $L$ from being decomposed into a direct product of two nontrivial sublattices.)

(d) FinItE-Dimensionality. There is a positive integer $n$ such that any strictly descending chain $x_{1}>x_{2}>\cdots>x_{k}$ of elements of $L$ has at most $n+1$ terms.

From this perspective, the classical coordinatization theorem of projective geometry [21, Chapter VI], [1, p. 302] states that with a few identifiable exceptions (corresponding to non-Desarguesian projective planes), every complemented, modular, irreducible, finite-dimensional lattice is isomorphic to the lattice of subspaces of a finite-dimensional vector space over a division ring. There is an alternate form of this conclusion, in terms of right ideals in matrix rings, which von Neumann found more convenient for generalization. Namely, the lattice of subspaces of an $n$-dimensional vector space over a division ring $D$ is isomorphic to the lattice of principal (i.e., singly-generated) right ideals in the ring of all $n \times n$ matrices over $D$. Thus, his model was a theorem stating that certain lattices are isomorphic to lattices of principal right ideals in certain rings.

For rings in general, the collection of principal right ideals need not form a lattice, let alone a complemented lattice; hence, von Neumann was led to the class of regular rings, which may be described as those rings in which the collection of principal right ideals does form a lattice under the obvious lattice operations. Precisely, he defined a regular ring to be a ring $R$ such that for any element $x \in R$ there is another element $y \in R$ satisfying $x y x=x$ [24, Definition 4]. The most immediate consequence of this condition is that $x y$ is an idempotent (that is, $(x y)^{2}=x y$ ) which generates the same principal right ideal as $x$. As a result, the complementary idempotent $1-x y$ generates a principal right ideal which is a complement for the principal right ideal generated by $x$. With a bit more computation, von Neumann showed that in a regular ring $R$, the sum and the intersection of finitely many principal right ideals are also principal right ideals. This suffices to make the collection of principal right ideals of $R$ into a lattice (with sums for least upper bounds and intersections for greatest lower bounds), with complements as above. In addition, modularity is easily checked.

Thus in any regular ring, the collection of principal right ideals forms a complemented modular lattice. In a striking tour-de-force, von Neumann proved a nearly complete converse: with certain exceptions (generalizing the non-Desarguesian exceptions in projective geometry), every complemented modular lattice is isomorphic to the lattice of principal right ideals of a regular ring, and the regular ring is unique up to isomorphism [25, Part II, Theorem 14.1]. (This regular ring is said to coordinatize the given complemented modular lattice.)

II. Von Neumann algebras, continuous geometries, and continuous regular rings. The complemented modular lattices of most interest to von Neumann occurred in his investigations with Murray of certain operator algebras. These algebras live inside the algebra $B(H)$ of all continuous linear transformations 
on a complex Hilbert space $H$. Von Neumann introduced a weak topology on $B(H)$ [22, p. 382]-namely the weakest topology under which the maps $T \mapsto(T(x), y)$ are continuous for all $x, y \in H$-and required his algebras to be closed in this topology. Also required was that the algebras be selfadjointalong with any operator $T$ in the algebra, the adjoint operator $T^{*}$ should also be included in the algebra. These requirements determine what von Neumann called rings of operators-weakly closed selfadjoint subalgebras of $B(H)$. Today these algebras are known as von Neumann algebras, or as $W^{*}$-algebras (where the " $W$ " abbreviates "weakly closed", while the "*" suggests "selfadjoint").

One of the major features of a von Neumann algebra $A$ is a large supply of projections. [A projection in $B(H)$ is an operator which projects onto a closed linear subspace of $H$ along the orthogonal complement. Algebraically, the projections in $B(H)$ are exactly the selfadjoint idempotents, i.e., operators $P$ satisfying $P=P^{*}=P^{2}$.] For example, if $T$ is any operator in $A$, then the projections onto the kernel of $T$ and onto the closure of the range of $T$ both lie in $A$.

The collection $P(A)$ of all projections in $A$ corresponds (by associating with any projection its range) to a subset of the lattice of closed linear subspaces of $H$. The inclusion relation among subspaces may be pulled back to define a natural partial order in $P(A)$, which is easily described algebraically: $P \leqslant Q$ if and only if $P=Q P$. It is a consequence of the weak closedness of $A$ that $P(A)$ is in fact a complete lattice-every subset of $P(A)$ has a least upper bound and a greatest lower bound in $P(A)$.

In the irreducible finite-dimensional case, $A$ is some $B(H)$, and $P(A)$ corresponds to the lattice of all subspaces of $H$, so that $P(A)$ is a complex projective geometry in the sense of Birkhoff and Menger. In this case, vector space dimension provides a natural dimension function on $P(A)$. If normalized by dividing by the dimension $n$ of $H$, this dimension function assumes the values $0,1 / n, 2 / n, \ldots, 1$.

Murray and von Neumann discovered certain infinite-dimensional cases where $P(A)$ possesses a dimension function satisfying most of the usual properties of (normalized) vector space dimension, except that the range of the dimension function is the entire unit interval [19, Theorem XII, p. 208]. As the dimension function here has a continuous range of values, the projection lattices $P(A)$ in these cases were named "continuous geometries" in contrast to the "discrete geometries" of the finite-dimensional case.

Like the projective geometries, these lattices are complemented, modular, and irreducible, although not finite-dimensional. They also have two other characteristic properties, which hold in projective geometries as easy consequences of finite-dimensionality. One is completeness, while the other is a weak infinite distributive law which von Neumann referred to as "continuity of the lattice operations", namely $x \wedge\left(\vee y_{\alpha}\right)=\bigvee\left(x \wedge y_{\alpha}\right)$ and $x \vee\left(\wedge y_{\alpha}\right)$ $=\wedge\left(x \vee y_{\alpha}\right)$ for any $x$ in the lattice and any linearly ordered subset $\left\{y_{\alpha}\right\}$ in the lattice. In keeping with von Neumann's terminology, any complete lattice satisfying this condition is now called a continuous lattice. 
Von Neumann used these key properties to abstractly define a continuous geometry as any complete, complemented, modular, continuous lattice which is irreducible but not finite-dimensional [23]. In current usage, reducibility and finite-dimensionality are both allowed, so that a continuous geometry is any complete, complemented, modular, continuous lattice. With this definition, the projection lattice $P(A)$ of a von Neumann algebra $A$ is a continuous geometry if and only if $A$ is finite in the technical sense that $x x^{*}=1$ always implies $x^{*} x=1$. (There is a natural family of extended real-valued generalized dimension functions definable on $P(A)$, and $A$ is finite in this sense exactly when these dimension functions all have finite bounded values.)

Because the interest in von Neumann's lattice-theoretic work was focussed mainly on continuous geometries, most of the interest in regular rings was for a number of years concentrated on those regular rings which coordinatize continuous geometries. These rings, that is, regular rings for which the lattice of principal right ideals is a continuous geometry, are known as continuous regular rings, and quite a complete structure theory has developed for them parallel to the structure theory for von Neumann algebras [12, Chapters 9-13].

For a finite von Neumann algebra $A$, von Neumann's coordinatization theorem yields a continuous regular ring $R$ whose lattice of principal right ideals is isomorphic to $P(A)$. In addition, the natural anti-isomorphism of $P(A)$ onto itself (via the map $P \mapsto 1-P$ ) induces an involution ${ }^{*}$ on $R$ (i.e., an anti-automorphism of period two) such that every principal right ideal of $R$ is generated by a projection; thus $R$ becomes what is called a ${ }^{*}$-regular ring. Actually, not only the lattice structure of $R$ is connected with $A$, but also the ring structure, for Murray and von Neumann showed that $R$ can be constructed directly by enlarging $A$ to contain certain discontinuous operators defined on dense linear subspaces of the Hilbert space on which $A$ acts [19, Theorem XV, p. 229], [25, pp. 26-27]. The involution on $R$ is an extension of the adjoint operation $T \mapsto T^{*}$ on $A$, and the only projections in $R$ are those already in $A$. Thus the projection lattices $P(A)$ and $P(R)$ coincide, providing a very direct isomorphism of $P(A)$ onto the lattice of principal right ideals of $R$ (namely the map associating to any $P \in P(A)$ the principal right ideal of $R$ generated by $P$ ).

III. $A W^{*}$-algebras and continuous regular rings. In order to deal with projections in von Neumann algebras on a basis intrinsic to the algebras (i.e., without reference to the Hilbert spaces on which they act), Kaplansky abstracted the basic properties to define $A W^{*}$-algebras $[17$, p. 236] (the name was chosen to suggest "abstract $W^{*}$-algebras"). These algebras are first of all $C^{*}$-algebras, that is, Banach algebras with an involution ${ }^{*}$ in which $\left\|x x^{*}\right\|=$ $\|x\|^{2}$. Then an $A W^{*}$-algebra is a $C^{*}$-algebra $A$ such that for any subset $X \subseteq A$, the right annihilator ideal

$$
\{a \in A \mid x a=0 \text { for all } x \in X\}
$$

is a principal right ideal generated by a projection.

As in the von Neumann algebra case, the collection $P(A)$ of projections in an $A W^{*}$-algebra $A$ forms a complete lattice, and $P(A)$ is a continuous 
geometry if and only if $A$ is finite in the technical sense. In this case, there is again the question of exhibiting the regular ring which coordinatizes $P(A)$. By using an abstract version of the Murray-von Neumann construction, Berberian showed in [2] that a finite $A W^{*}$-algebra $A$ is always contained in a continuous *-regular ring $R$ such that $R$ has no new projections beyond those in $A$.

Since the coordinatizing regular ring has proved useful in investigating continuous geometries, this might also be expected in investigating $A W^{*}$-algebras. However, except in one case, the axioms of $A W^{*}$-algebras have proved strong enough to deal efficiently with the questions that have arisen, without needing to use the associated regular rings. The exceptional case occurred when Berberian proved that the algebra of $n \times n$ matrices over any $A W^{*}$-algebra is again an $A W^{*}$-algebra [3, Theorem, p. 43]. The proof easily reduces to the case of a finite $A W^{*}$-algebra $A$, which has an associated regular ring $R$, and some of the steps of the proof were worked out within the ring of $n \times n$ matrices over $R$, which is a tractable ring because of the general result that the ring of $n \times n$ matrices over any *-regular ring with a positive definite involution is again a *-regular ring. For another illustration of the possible uses of the regular rings associated with finite $A W^{*}$-algebras, consider the following open problem.

Let $A$ be a finite $A W^{*}$-algebra which is infinite-dimensional and also a simple algebra (i.e., the only ideals of $A$ are $(0)$ and $A$ ). The $2 \times 2$ matrix algebra $M_{2}(A)$ is, using the appropriate norm and the *-transpose involution, also a simple, infinite-dimensional, finite $A W^{*}$-algebra. In the most tractable case, namely when $A$ can be generated (as an $A W^{*}$-algebra) by the union of an ascending sequence of finite-dimensional simple subalgebras, it is known that $M_{2}(A)$ is isomorphic to $A$. The open problem is whether $M_{2}(A)$ must always be isomorphic to $A$.

Now the regular ring $R$ associated with $A$ is a simple, continuous, *-regular ring which is not artinian (i.e., there are arbitrarily long descending chains of right ideals in $R$ ). With the *-transpose involution, the $2 \times 2$ matrix ring $M_{2}(R)$ has the same properties. The algebras $A$ and $M_{2}(A)$ can be canonically identified inside $R$ and $M_{2}(R)$ as the subrings of "bounded elements", as in [4, Theorem 1, p. 245]. Consequently, any involution-preserving isomorphism of $M_{2}(R)$ onto $R$ would restrict to an isomorphism of $M_{2}(A)$ onto $A$.

Thus we obtain a purely ring-theoretic problem: if $R$ is a nonartinian, simple, continuous, *-regular ring, are $M_{2}(R)$ and $R$ isomorphic (as *-rings)? A positive answer to this problem would then yield a positive answer to the $A W^{*}$-algebra problem outlined above.

IV. Rickart $C^{*}$-algebras and $\boldsymbol{\aleph}_{0}$-continuous regular rings. During the trend of axiomatization that led to $A W^{*}$-algebras, Rickart introduced a weak version of $A W^{*}$-algebras which he called $B_{p}^{*}$-algebras [20, Definition 2.1], but which are now known as Rickart $C^{*}$-algebras. Such algebras are $C^{*}$-algebras in which it is only assumed that the right annihilator ideal of any single element (rather than the right annihilator ideal of any subset, as in the definition of $A W^{*}$-algebras) is a principal right ideal generated by a projection. In an 
appendix to his development of $A W^{*}$-algebras [17, p. 249], Kaplansky showed that a few of the most basic $A W^{*}$-algebra proofs carry over to Rickart $C^{*}$-algebras, but then gave an example showing that one of the most widely useful properties of $A W^{*}$-algebras-central comparability-does not always hold for Rickart $C^{*}$-algebras. He then pessimistically concluded: "The pathology shown by this example indicates that it is probably fruitless to pursue the theory of [Rickart $C^{*}$-algebras]."

However, recent work has shown that Rickart $C^{*}$-algebras are not so intractable after all, and many reasonable analogs of $A W^{*}$-algebra results do in fact hold. The breakthrough was Handelman's discovery of an appropriate analog to the regular ring constructions of Murray-von Neumann and Berberian, as follows. In any finite Rickart $C^{*}$-algebra $A$, the projection lattice $P(A)$ satisfies most of the axioms of a continuous geometry, except that $P(A)$ is only countably complete (only countable subsets of $P(A)$ must have least upper bounds and greatest lower bounds), and the "continuity" of the lattice operations is only required to hold for countable subsets. Such a lattice is called an $\boldsymbol{\aleph}_{0}$-continuous geometry, and any regular ring coordinatizing such a lattice is called an $\aleph_{0}$-continuous regular ring. Handelman showed that any finite Rickart $C^{*}$-algebra $A$ is canonically embedded in an $\aleph_{0}$-continuous *-regular ring $R$ such that $R$ and $A$ have the same projections (thus $R$ naturally coordinatizes $P(A)$ ) [15, Theorem 2.1$]$.

Handelman's construction makes it possible to apply the method which was neglected in the $A W^{*}$-algebra case, namely the transfer of problems concerning a finite Rickart $C^{*}$-algebra $A$ to corresponding questions concerning the associated $\aleph_{0}$-continuous *-regular ring $R$. Here is a sample of results which have been proved in this manner. In each case, the key step is the application to $R$ of a corresponding result about $\boldsymbol{\aleph}_{0}$-continuous regular rings, which we quote parenthetically. Any element $a \in A$ has a right projection (the smallest projection $p \in P(A)$ for which $a p=a$ ) and a corresponding left projection. Kaplansky conjectured that the right and left projections of any element of $A$ must be equivalent [17, p. 249], and Handelman proved this in [15, Theorem 4.1]. (Whenever two idempotents $e$ and $f$ in an $\aleph_{0}$-continuous regular ring are equivalent, the complementary idempotents $1-e$ and $1-f$ are equivalent also [15, Theorem 3.2].) Handelman also proved that for every maximal ideal $M$ of $A$, the quotient algebra $A / M$ is a finite $A W^{*}$-algebra [15, Theorem 4.3]. (Any quotient ring of an $\aleph_{0}$-continuous regular ring modulo a maximal ideal is a continuous regular ring [15, Corollary 3.2].) Continuing in this direction, Handelman, Higgs, and Lawrence showed that the intersection of the maximal ideals of $A$ is zero, leading to an embedding of $A$ into a finite $A W^{*}$-algebra [16, Theorem 3.1]. (In any $\aleph_{0}$-continuous regular ring, the intersection of the maximal ideals is zero [16, Theorem 2.3].) Goodearl, Handelman, and Lawrence proved that if $A$ has no finite-dimensional representations, then $A$ is isomorphic to an $n \times n$ matrix algebra for every positive integer $n$ [13, Theorem III.16.8]. (If an $\aleph_{0}$-continuous regular ring has no simple artinian homomorphic images, then it is an $n \times n$ matrix ring for every $n[13$, Theorem II.15.4].) 
V. Approximately finite-dimensional $C^{*}$-algebras and ultramatricial regular rings. Among general $C^{*}$-algebras, there is a reasonably manageable class which still exhibits many of the general phenomena, namely the approximately finite-dimensional (or $A F$ ) $C^{*}$-algebras introduced by Bratteli [6, Definition 1.1]: these are the $C^{*}$-algebras possessing a norm-dense subalgebra which is the union of an increasing sequence of finite-dimensional sub- $C^{*}$-algebras. Bratteli showed that such a $C^{*}$-algebra and such a dense subalgebra determine each other, in the following manner: If $A$ and $A^{\prime}$ are $C^{*}$-algebras with norm-dense subalgebras $R$ and $R^{\prime}$ which are unions of increasing sequences of finite-dimensional sub- $C^{*}$-algebras, then $A$ and $A^{\prime}$ are isomorphic as $C^{*}$-algebras if and only if $R$ and $R^{\prime}$ are isomorphic as *-algebras [6, Theorem 2.7]. Moreover, $R$ and $R^{\prime}$ are isomorphic as *-algebras if and only if they are isomorphic just as algebras [8, Appendix].

Since finite-dimensional $C^{*}$-algebras are semisimple and thus ${ }^{*}$-regular, so are increasing unions of them. Thus there is a class of *-regular algebras whose isomorphism classes determine the isomorphism classes of the class of $A F C^{*}$-algebras. The analogous algebras may be defined over any field $F$ (where they are merely regular as opposed to *-regular): an ultramatricial $F$-algebra is any $F$-algebra which is a union of an increasing sequence of finite-dimensional subalgebras, each of which is a finite direct product of full matrix algebras over $F$.

Ultramatricial algebras were completely classified by Elliott in terms of an invariant which may be expressed as a generating base of a positive cone of a partially ordered "dimension group" [8, Theorems 4.3, 5.1, 5.3]. An equivalent, more compact, classification is in terms of the Grothendieck group, $K_{0}$, of the algebra. This is an abelian group which can be defined for any ring $R$, with generators corresponding to the isomorphism classes of finitely generated projective right $R$-modules, and with an addition operation induced from the direct sum operation on the modules. There is a natural translationinvariant pre-order relation on $K_{0}(R)$, which is a partial order in the case of ultramatricial algebras, and there is also a natural "order-unit" in $K_{0}(R)$, given by the generator corresponding to the canonical free right $R$-module of rank one.

In these terms, Elliott's classification may be expressed as follows. First, if $R$ and $R^{\prime}$ are ultramatricial $F$-algebras, then $R$ and $R^{\prime}$ are isomorphic as $F$-algebras if and only if $K_{0}(R)$ and $K_{0}\left(R^{\prime}\right)$ are isomorphic as partially ordered abelian groups with order-unit [12, Theorem 15.26]. Second, for an ultramatricial algebra $R$, the partially ordered abelian group $K_{0}(R)$ is a direct limit of a sequence of finite rank free abelian groups with the direct product ordering, and all such groups do arise [12, Theorem 15.24]. A much more convenient description of these groups has recently been proved by Effros, Handelman, and Shen [7, Theorem 2.2]: A partially ordered abelian group $G$ is isomorphic to $K_{0}$ of an ultramatricial $F$-algebra if and only if

(a) $G$ is countable.

(b) $G$ has an order-unit.

(c) If $x \in G$ and $n x \geqslant 0$ for some positive integer $n$, then $x>0$.

(d) If $x_{1}, x_{2}, y_{1}, y_{2} \in G$ with $x_{i} \leqslant y_{j}$ for each $i, j$, then there exists $z \in G$ such that $x_{i} \leqslant z \leqslant y_{j}$ for each $i, j$. 
VI. Regular rings and Choquet simplices. The continuous geometries which Murray and von Neumann first investigated, namely the projection lattices of certain von Neumann algebras, came equipped with dimension functions. Von Neumann later constructed dimension functions on arbitrary irreducible continuous geometries, and translated these dimension functions into dimension-like functions on the coordinatizing regular rings. Specifically, he defined a rank function [25, Part II, p. 161] on a regular ring $R$ to be a map $N: R \rightarrow[0,1]$ such that

(a) $N(x)=0$ if and only if $x=0$,

(b) $N(1)=1$,

(c) $N(x y) \leqslant N(x)$ and $N(x y) \leqslant N(y)$,

(d) $N(e+f)=N(e)+N(f)$ for orthogonal idempotents $e$ and $f$.

Such functions he considered to be a generalization of normalized matrix rank.

When $R$ coordinatizes an irreducible continuous geometry (the case von Neumann was mainly interested in), there is a unique rank function on $R$, and condition (a) of the definition is essentially just a consequence of irreducibility. For more general regular rings, however, condition (a) is too restrictive, hence it becomes appropriate to consider pseudo-rank functions [9, p. 269], namely maps $N: R \rightarrow[0,1]$ satisfying conditions (b), (c), (d).

In any regular ring $R$, the collection $\mathbf{P}(R)$ of all pseudo-rank functions on $R$ may be viewed as a subset of the real linear topological space $\mathbf{R}^{R}$ of all real-valued functions on the set $R$ (where $\mathbf{R}^{R}$ is given the product topology). Goodearl showed that $\mathbf{P}(R)$ is a compact convex subset of $\mathbf{R}^{R}[\mathbf{9}, \mathrm{pp} .270$, 273], and that $P(R)$ is in fact a Choquet simplex [10, Corollary 3.6]. (Choquet simplices are a standard infinite-dimensional generalization of classical finite-dimensional simplices.) The structure of $\mathbf{P}(R)$ can be investigated as an invariant of the structure of $R$. For instance, $\mathbf{P}(R)$ carries enough information to determine the decomposition properties of certain completions of $R$, as follows.

Von Neumann noted that any rank function $N$ on a regular ring $R$ induces a metric $\delta_{N}$ on $R$, where $\delta_{N}(x, y)=N(x-y)$ [25, Part II, pp. 161-162], and in fact if $N$ is a pseudo-rank function, the same argument shows that $\delta_{N}$ is a pseudo-metric. The completion of $R$ with respect to $\delta_{N}$ is a ring, called the $N$-completion of $R$; Halperin showed that this $N$-completion is a regular ring [14, Theorem 3.7], and later Goodearl showed that it is actually a continuous regular ring [9, Corollary 15]. In [9, Theorems 19, 22], Goodearl showed that the $N$-completion is a direct product of $t(=1,2, \ldots, \infty)$ simple rings if and only if $N$ is a positive convex combination of $t$ distinct extreme points of $\mathrm{P}(R)$. Moreover, the Boolean algebra of central idempotents in the $N$-completion is naturally isomorphic to a certain lattice of faces in $\mathbf{P}(R)$ related to $N$ [11, Theorem 3.7].

Another case in which $\mathbf{P}(R)$ has proved particularly useful is that of an $\aleph_{0}$-continuous regular ring $R$, in relation to the partially ordered Grothendieck group $K_{0}(R)$. Over any regular ring $R$, every pseudo-rank function induces a dimension function on the lattice of principal right ideals of $R$, which extends to a dimension function on the class of finitely generated 
projective right $R$-modules. This latter dimension function in turn induces an order-preserving homomorphism of $K_{0}(R)$ into $\mathbf{R}$. Thus we obtain a map of $\mathbf{P}(R)$ into the space of order-preserving real-valued homomorphisms on $K_{0}(R)$. Taking the dual viewpoint, each element of $K_{0}(R)$ induces a map from $\mathbf{P}(R)$ to $\mathbf{R}$, which is affine and continuous. This leads to a natural order-preserving homomorphism of $K_{0}(R)$ into the space $\operatorname{Aff}(\mathrm{P}(R))$ of all affine continuous real-valued functions on $\mathbf{P}(R)$.

When $R$ is an $\aleph_{0}$-continuous regular ring, Goodearl, Handelman, and Lawrence have shown that this natural map of $K_{0}(R)$ into $\operatorname{Aff}(P(R))$ is an order-embedding, and its image is precisely described [13, Theorem II.15.1]. In particular, if $R$ has no simple artinian homomorphic images, then $K_{0}(R)$ is isomorphic (as a partially ordered abelian group) to $\operatorname{Aff}(\mathbf{P}(R))$ itself $[13$, Corollary II.15.2]. The consequence that $K_{0}(R)$ is, in this case, a rational vector space, leads directly to the result that $R$ is an $n \times n$ matrix ring for every positive integer $n$, which in turn leads to the corresponding result for finite Rickart $C^{*}$-algebras, mentioned above.

\section{REFERENCES}

1. R. Baer, Linear algebra and projective geometry, Academic Press, New York, 1952.

2. S. K. Berberian, The regular ring of a finite $A W^{*}$-algebra, Ann. of Math. (2) 65 (1957), 224-240.

3. __ $N \times N$ matrices over an $A W^{*}$-algebra, Amer. J. Math. 80 (1958), 37-44.

4. _ Baer *-rings, Die Grundlehren der Math. Wissenschaften, Band 195, SpringerVerlag, Berlin and New York, 1972.

5. G. Birkhoff, Combinatorial relations in projective geometries, Ann. of Math. (2) 36 (1935), $743-748$.

6. O. Bratteli, Inductive limits of finite-dimensional $C^{*}$-algebras, Trans. Amer. Math. Soc. 171 (1972), 195-234.

7. E. G. Effros, D. E. Handelman, and C.-L. Shen, Dimension groups and their affine representations, Amer. J. Math. 102 (1980), 385-407.

8. G. A. Elliott, On the classification of inductive limits of sequences of semisimple finite-dimensional algebras, J. Algebra 38 (1976), 29-44.

9. K. R. Goodearl, Simple regular rings and rank functions, Math. Ann. 214 (1975), 267-287.

10. __ Completions of regular rings, Math. Ann. 220 (1976), 229-252.

11. __ Completions of regular rings. II, Pacific J. Math. 72 (1977), 423-459.

12. __ Von Neumann regular rings, Pitman, London, 1979.

13. , D. E. Handelman, and J. W. Lawrence, Affine representations of Grothendieck groups and applications to Rickart $C^{*}$-algebras and $\aleph_{0}$-continuous regular rings,Mem. Amer. Math. Soc. No. 234 (1980).

14. I. Halperin, Regular rank rings, Canad. J. Math. 17 (1965), 709-719.

15. D. Handelman, Finite Rickart $C^{*}$-algebras and their properties, Studies in Analysis, Advances in Math. Supplementary Studies, Vol. 4, 1979, pp. 171-196.

16. D. Higgs, and J. Lawrence, Directed abelian groups, $\aleph_{0}$-continuous rings, and Rickart $C^{*}$-algebras, J. London Math. Soc. 21 (1980), 193-202.

17. I. Kaplansky, Projections in Banach algebras, Ann. of Math. (2) 53 (1951), 235-249.

18. K. Menger, New foundations of projective and affine geometry, Ann. of Math. (2) 37 (1936), 456-482.

19. F. J. Murray and J. von Neumann, On rings of operators, Ann. of Math. (2) 37 (1936), 116-229.

20. C. E. Rickart, Banach algebras with an adjoint operation, Ann. of Math. (2) 47 (1946), 528-550. 
21. O. Veblen and J. W. Young, Projective geometry, Vol. I, Ginn and Co., Boston, 1910.

22. J. von Neumann, Zur Algebra der Funktionaloperationen und Theorie der normalen Operatoren, Math. Ann. 102 (1929), 370-427.

23. Continuous geometry, Proc. Nat. Acad. Sci. U.S.A. 22 (1936), 92-100.

24. __ On regular rings, Proc. Nat. Acad. Sci. U.S.A. 22 (1936), 707-713.

25. Lectures on continuous geometry (Planographed notes, Institute for Advanced Study), Edwards Brothers, Ann Arbor, Michigan, 1937.

Department of Mathematics, University of Utah, Salt Lake City, Utah 84112 